\title{
The irreplaceable role of ubiquitous cosmic rays in the space chemistry: from the origin of complex species in interstellar molecular clouds to the ozone depletion in the atmospheres of Earth-like planets
}

\author{
Ararat Yeghikyan* \\ Byurakan Astrophysical Observatory, Byurakan, Aragatsotn Province, Armenia
}

\begin{abstract}
A review is given of low-energy cosmic rays $(1 \mathrm{MeV}-10 \mathrm{GeV})$, which play an important role in the physics and chemistry of interstellar medium of our Galaxy. According to the generally accepted theory of star formation, cosmic rays penetrate into molecular clouds and ionize the dense gaseous medium of star formation centers besides due to a process of ambipolar diffusion they establish a star formation time scale of about 100-1000 thousand years. The source of cosmic rays in the Galaxy are supernovae remnants where diffusion acceleration at the shock front accelerates particles up to energies of $10^{15} \mathrm{eV}$. Being the main source of ionization in the inner regions of molecular clouds, cosmic rays play a fundamental role in the global chemistry of clouds, triggering the entire chain of ion-molecular reactions that make it possible to obtain basic molecules. The review also noted the importance of cosmic rays in atmospheric chemistry: playing a significant role in the formation of nitric oxide, especially with an increase in the flux, they cause a decrease in the concentration of ozone in the atmosphere with all climatic consequences.
\end{abstract}

Keywords: cosmic rays - sources - molecular cloud chemistry - origin of complex species - nitric oxide in terrestrial atmosphere - ozone content

\section{Introduction}

Cosmic rays (CR) were discovered in 1911 by the German physicist V. Hess in 1912 in a series of balloon experiments showing an increase of the background radiation with height. The history of the discovery with interesting details can be found in Ginzburg (1996). Currently, cosmic rays are considered to be one of the most important component of the galactic interstellar medium, along with gas and dust which play an important role in the whole energy balance in the Galaxy, and regulate important processes in molecular clouds associated with star formation, initiation of astrochemical reactions, the origin of complex organic species, etc. CRs are nuclei of atoms in the energy range of $\mathrm{MeV}-\mathrm{GeV}$ and higher, starting with protons and $\alpha$ particles. The remaining nuclei are observed in accordance with their cosmic abundance, excepted nuclei formed during CR transport in the Galaxy. CRs are observed up to energies of $10^{20} \mathrm{eV}$, which are clearly extragalactic and do not, by the way, have a generally accepted explanation of the source of origin. In this review, we restrict our discussion about CR with energies up to $10^{15} \mathrm{eV}$ arising in galactic sources through generally accepted acceleration mechanisms, one of which (diffusive) will be considered in sufficient details. We outline the important role of CR in determining the star formation time scale, and finally, analyze the possibility of formation complex compounds in molecular clouds initiated by CR, and some other questions, like ozone behavior in atmospheres of exoplanets (having earth's atmosphere as an example), also influenced by CR. Other questions of CR physics, related, for example, to CR transport in the Galaxy, accompanying nuclear reactions, etc., will remain outside our consideration.

*ayarayeg@gmail.com 


\section{Cosmic rays in general: sources, acceleration and interaction with matter}

The main sources of CR in the Galaxy are supernova remnants (SNII) Schlickeiser (2002), Longair (2011), Bykov et al. (2018). As is known, in a supernova explosion, namely, during a nuclear collapse, it is possible to release energy of the order of $\sim G M^{2} / R$, up to $10^{53}$ erg during a mass collapse of $M \sim M_{\odot}$ into a region with the size of $R \sim 10^{6} \mathrm{~cm}$, that is, with the formation of a neutron star. In this case, according to observational data, of the order of $\varepsilon \sim 10-30 \%$ of the kinetic energy of the expansion of the remnant (so, $E_{k i n} \sim M V^{2} \sim 10^{51} \mathrm{erg}$ ), with an expansion velocity of $10^{9} \mathrm{~cm} / \mathrm{s}$ of the mass of $M_{\odot}$ ) goes into the energy of CR particles, up to $10^{15} \mathrm{eV}$ per proton Longair (2011), Bykov et al. (2018). We recall, for completeness, that the excess energy of the explosion is carried away by neutrinos that practically do not interact with the medium.

Returning to CR, it should be emphasized that in order to ensure the observed average CR flux (at proton energies $\sim 1 \mathrm{MeV}$ moving with a velocity $V_{p}$ ) in the Galaxy of the order of $1 . \cdot 10^{-4}$ particles $\cdot \mathrm{cm}^{-2} \cdot \mathrm{s}^{-1} \cdot \mathrm{sr}^{-1} \cdot \Delta \mathrm{E}^{-1}$ Stone et al. (2005), Scherer et al. (2008), and for which one can write an approximation formula

$$
J_{p}=\varepsilon \cdot \frac{M V^{2} \cdot V_{p}}{4 \pi \cdot \tau \cdot V_{G} \cdot \Delta E},
$$

it is necessary to have a supernova explosion frequency (of type II) in the Galaxy, $\tau \sim 1$ for 50 years Diehl et al. (2006), more precisely, $\tau=1.9 \pm 1.1$ per century), per unit volume. For $V_{G}$ in the form of a disk with a radius of $15 \mathrm{kpc}$ and a height of $10 \mathrm{pc}$, we get just the value of $J_{p}$ for $\Delta E \sim 1$ $\mathrm{MeV}, J_{p}=4 \cdot 10^{-4}$ particles $\cdot \mathrm{cm}^{-2} \cdot \mathrm{s}^{-1} \cdot \mathrm{sr}^{-1} \cdot \Delta \mathrm{E}^{-1}$, which, in order of magnitude, coincides with the above observed value by Voyager 1 in the vicinity of the Sun Stone et al. (2005), if $\varepsilon=0.1$. Then, for the CR density in the energy range $E \sim 1 \mathrm{MeV}$, we have $\varrho(C R) \cdot \Delta E=4 \pi J_{p} / V_{p} \approx 1 \mathrm{eV} / \mathrm{cm}^{3}$, which coincides with the densities of thermal $\left(\sim n k T_{e}\right)$, magnetic $\left(\sim H^{2} / 8 \pi\right)$ and internal (in this case directional: $\sim \rho v^{2}$ ) gas flow energies of the interstellar medium (ISM) of the Galaxy (Dopita \& Sutherland (2003)). Interestingly, it is also coincides with the ISM UV radiation energy $\left(\sim \rho_{\nu} \sim F_{\nu} / c\right.$, where $F_{\nu}=4 \pi J_{\lambda} \lambda=4 \cdot 10^{-2} \mathrm{erg} \cdot \mathrm{cm}^{-2} \cdot \mathrm{s}^{-1}$ ) at $\lambda=5000 \AA$ Mathis et al. (1983), so again $\rho_{\nu} \sim 10^{-12}$ $\mathrm{erg} / \mathrm{cm}^{3}=1 \mathrm{eV} / \mathrm{cm}^{3}$.

\section{The role of $\mathrm{CR}$ in star formation timescale}

According to the generally accepted point of view stars are formed as a result of contraction of interstellar molecular clouds under the Jeans condition, that is, at the excess of gravitational forces over the total forces of internal gas - thermal, magnetic and turbulent pressures. However, the details and degrees of impact of the latter two are not entirely clear in the case of the formation of large mass stars Bodenheimer (2011). On the other hand, it is known that during the formation of smallmass stars, magnetic pressure contributes to the process of controlling the contraction of the cloud. The theory of the process is well known Bodenheimer (2011), in particular, it is known that the mentioned magnetic and turbulent pressure forces may decay much earlier than the cloud compresses with the formation of a protostar. The fact is that the force of magnetic pressure, which counteracts compression, weakens over time due to the phenomenon of ambipolar diffusion, which leads to field reduction: in reality, a key process is a redistribution of magnetic fluxes over the cloud volume but not field reduction Bodenheimer (2011). As is mentioned above there is an ion drift with respect to the neutrals, which leads to such effects.

To estimate the time of free fall of the cloud mass $M$ at a distance $R$ in the absence of pressure forces we take $t_{f f}=R / v_{s}$ where $v_{s}$ is the speed of sound in the cloud medium under isothermal compression with a temperature $T_{c}=10 \mathrm{~K}$. Actually, the free fall velocity is $v_{f f}>v_{s}$, but since we need just the lower limit of the compression time, we use $v_{s}=\sqrt{k T_{c} / \mu m_{p}} \approx 0.3 \mathrm{~km} / \mathrm{s}$. For the protostellar cloud under the Jeans criterion, with $R_{c} \sim 10^{17}-10^{18} \mathrm{~cm}$, then $t_{f f} \approx 10^{5}-10^{6}$ years.

Let us now estimate the time of a significant weakening of the magnetic field due to the phenomenon of ambipolar diffusion. As is known, ambipolar diffusion is caused by the displacement of ions relative 
to neutrals in the inner regions of the clouds, induced by a magnetic field, and the field itself weakens mainly due to the redistribution of the magnetic flux in the cloud (Bartelmann, 2013). To estimate the characteristic time of the phenomenon, it is necessary to have an estimate of the rate of ion displacement relative to neutrals, $v_{d}$. The process is determined by collisions of particles (ions with neutrals) in a plasma with a magnetic field strength $B$ : the final result is given by (Bartelmann, 2013)

$$
v_{d} \approx \frac{B^{2}}{4 \pi \gamma \rho_{i} \rho_{n} R},
$$

where $R$ is the characteristic radius, $\rho_{i}$ and $\rho_{n}$ are the densities of ions and neutrals, and $\gamma$ is the so-called coefficient of friction, which actually determines the process rate. In the approximation of small relative velocities, $\gamma \sim 10^{14} \mathrm{~cm}^{3} \mathrm{~g}^{-1} \mathrm{~s}^{-1}$ Padmanabhan (2000). The diffusion rate can be related to the degree of ionization $x$, equating the forces of gravity and friction (caused by particle collisions) acting on the unit volume:

$$
\frac{G M \rho}{R^{2}}=n_{i}\langle\sigma \cdot v\rangle n\left(H_{2}\right) m\left(H_{2}\right) v_{d}
$$

Then

$$
v_{d}=\frac{4 \pi}{3} \frac{G \rho R}{n_{i}\langle\sigma \cdot v\rangle} \approx R \frac{10^{-8}}{x}
$$

and,

$$
t_{A D}=\frac{R}{v_{d}}=5 \cdot 10^{5} \frac{x}{10^{-8}} .
$$

The degree of ionization in the inner regions of the clouds is small, $x \sim 10^{-8}$ (see below), so the ambipolar diffusion is able to weaken the magnetic field to a degree that does not prevent further compression of the cloud in general, and the formation of a protostar, in particular. Since the cloud is fragmented during compression Padmanabhan (2000), it is the diffusion of the magnetic field in the cloud (as well as the turbulent pressure decay process not considered here) that determines the temporal and spatial scales of star formation.

\section{The degree of ionization in molecular clouds}

Cosmic rays play an active role in the regulation of star formation, through the ionization of the inner parts of the cloud. The degree of ionization is usually not very high; it can be estimated as follows. Molecular hydrogen is ionized first, and two channels of interaction are possible:

$$
\begin{gathered}
\mathrm{H}_{2}+C R \rightarrow \mathrm{H}_{2}^{+}+e \\
\rightarrow \mathrm{H}^{+}+\mathrm{H}+e,
\end{gathered}
$$

with ionization rates $\zeta=1.2 \cdot 10^{-17} \mathrm{~s}^{-1}$ and $\zeta=2.9 \cdot 10^{-19} \mathrm{~s}^{-1}$, respectively. Then a reaction occur with $\mathrm{H}_{2}$, which velocity is $k$ (in $\mathrm{cm}^{3} / \mathrm{s}$ ),

$$
\mathrm{H}_{2}^{+}+\mathrm{H}_{2} \rightarrow \mathrm{H}_{3}^{+}+\mathrm{H}
$$

with $k=2.1 \cdot 10^{-9} \mathrm{~cm}^{3} / \mathrm{s}$, and

$$
\mathrm{H}_{3}^{+}+e \rightarrow \mathrm{H}_{2}+\mathrm{H},\left(k^{\prime} \approx k\right) .
$$

Accordingly, since the degree of ionization in molecular clouds is determined by the rate of molecular hydrogen ionization by $\mathrm{CR}$, and $\mathrm{CO}$ molecules and $\mathrm{O}$ atoms are very abundant, $\mathrm{H}_{3}^{+}$most often reacts with them via

$$
\begin{gathered}
\mathrm{H}_{3}^{+}+\mathrm{CO} \rightarrow \mathrm{HCO}^{+}+\mathrm{H}_{2}, \\
\mathrm{H}_{3}^{+}+\mathrm{O} \rightarrow \mathrm{OH}^{+}+\mathrm{H}_{2},
\end{gathered}
$$


moreover, $\mathrm{OH}^{+}$immediately hydrogenates (by adding $\mathrm{H}_{2}$ ) to form $\mathrm{OH}_{3}^{+}$. Further, $\mathrm{HCO}^{+}$and $\mathrm{OH}_{3}^{+}$ recombine with free electrons to form neutral molecules. Ultimately, for electron concentration we can write

$$
\frac{d[e]}{d t}=\zeta\left[\mathrm{H}_{2}\right]-k_{r}\left(\left[\mathrm{HCO}^{+}\right]+\left[\mathrm{OH}_{3}^{+}\right]\right)[e],
$$

where $k_{r}$ is the average value of the coefficient of electronic recombination $\mathrm{HCO}^{+}$and $\mathrm{OH}_{3}^{+}$with electrons $\left(k_{r}=2.5 \cdot 10^{-6} \mathrm{~cm}^{3} / \mathrm{s}\right.$ at $\left.10 \mathrm{~K}\right)$, and the square brackets indicate the concentrations. Since these two ions are the dominant carriers of a positive charge, we can define the condition of the charge balance in the form Tielens (2005)

$$
\left[\mathrm{HCO}^{+}\right]+\left[\mathrm{OH}_{3}^{+}\right]=[e] .
$$

Then, under stationary conditions, for the degree of ionization we have:

$$
\frac{e}{\mathrm{H}_{2}}=\sqrt{\frac{\zeta}{k_{r}\left[\mathrm{H}_{2}\right]}}=\frac{2.2 \cdot 10^{-6}}{\sqrt{\left[\mathrm{H}_{2}\right]}},
$$

whence it follows that the degree of ionization in dense, cold clouds is small, for example, $\sim 10^{-8}$ at $\left[\mathrm{H}_{2}\right]=10^{4} \mathrm{~cm}^{-3}$.

We emphasize here that if the value of the CR flux, $F(E)$ is known from observations, then the value of the ionization rate of molecular hydrogen by $\mathrm{CR}, \zeta$ can be estimated by the formula Jenniskens (1993)

$$
\zeta \sim F(E) \Delta(E) \sigma(E)
$$

where $\Delta(E)=1 \mathrm{MeV}$, and $\sigma(E)$ is the ionization cross section in $\mathrm{cm}^{2}$ (Fig. 1, where now $\Delta(E)=$ $1 \mathrm{eV})$. Since, say, $\sigma(5 \mathrm{MeV}) \sim 1.0 \cdot 10^{-17} \mathrm{~cm}^{2}$, and $F(5 \mathrm{MeV}) \sim 1.0$ particles $\cdot \mathrm{cm}^{-2} \cdot \mathrm{s}^{-1} \cdot \mathrm{sr}^{-1} \cdot \mathrm{MeV}^{-1}$, then $\zeta \sim 1.0 \cdot 10^{-17} \mathrm{~s}^{-1}$, which by order of magnitude coincides with observed values.

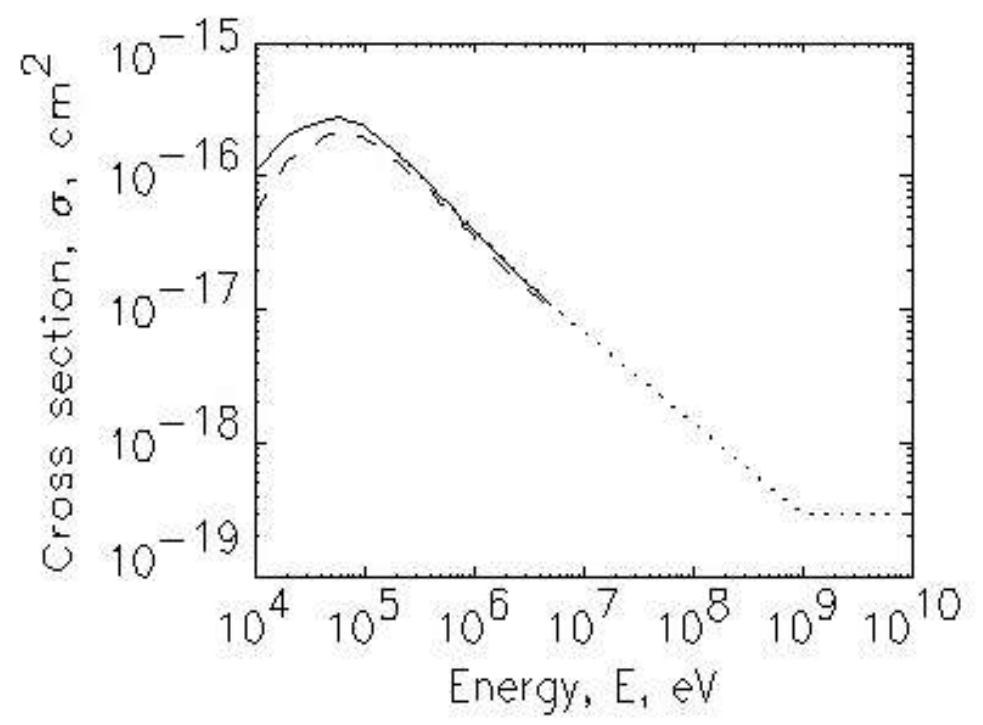

Figure 1. The dependence of the ionization cross section of $\mathrm{H}_{2}$ by protons on energy.

\section{On the diffusive acceleration of particles at the front of a shock wave}

Let us now turn to the possibility of particle acceleration under the conditions of interaction of expanding stellar material with the interstellar medium (or with the remnant of the previous outflow matter). It is clear that the definition of expanding stellar material includes not only the remnants of supernovae studied in detail in Longair (2011), but also stellar winds, including solar 
Scherer et al. (2008). At present, diffusive acceleration by the Fermi 1 mechanism at the front of a magnetohydrodynamic shock wave is considered as the most effective one for particles acceleration. This assumes the presence of a certain amount of energetic particles from superthermal ions entering the acceleration mode in the region of the internal shock front (such particles are known as injected).

Further, there are a large number of analytical and numerical models describing the intensities of energetic particles accelerated at the front of the shock wave and taking into account many features of the process, such as the formation of an energetic spectrum of particles, the orientation of the magnetic field vector relative to the normal to the front, sources of superthermal particles that are accelerated by the diffusive mechanism and transformed into the anomalous component of cosmic rays (ACR in the case of the heliosphere) Drury (1983), Ellison et al. (2005), Fahr \& Versvharen (2008), Fahr \& Versvharen (2009), Yeghikyan (2018b), Yeghikyan (2018a). These theories predict reasonable values of particle intensities consistent with direct observations of Voyager probes in the heliosphere. Therefore, the results of one such analytical theory describing the spectrum of particles under conditions of a parallel shock wave in the heliosphere Yeghikyan (2018a), and used for the conditions of WR nebulae Yeghikyan (2018b), will be presented below. Under the heliosphere, we are talking about the case when the protons of the solar wind are reflected from the front of a quasi-parallel magnetohydrodynamic shock wave upstream and are captured in the form of a group of superthermal particles entering into the diffusive acceleration process Fahr \& Verscharen (2016), Fahr \& Versvharen (2009). Here the question may arise about the similarity of the conditions of shock waves of the heliosphere and the WR nebulae, while nothing is known about the geometry of the magnetic fields of the latter. Here, we restrict ourselves to the references in Fahr \& Versvharen (2008), Scherer \& Fahr (2009), where it is shown that the manifestations of the quasi-parallel configuration of the shock wave in the heliosphere are very probable and significant, and we will mention the similarity with WR stars below.

Thus, in the case of a planar front, for the conditions of a quasiparallel shock, we have for the differential intensity of accelerated particles (in units of particles $\cdot \mathrm{cm}^{-2} \cdot \mathrm{s}^{-1} \cdot \mathrm{sr}^{-1} \cdot \mathrm{MeV}^{-1}$ ) Fahr \& Versvharen (2009):

$$
\Phi_{0}(E)=\frac{1}{4 \pi} \frac{\varepsilon_{1} n_{1} v_{01}}{\Lambda(s)} \frac{1}{2 E_{0}}\left(\frac{E}{E_{0}}\right)^{\frac{2-q}{2}},
$$

where $v_{01}=\sqrt{2 E_{0} / m} \cong 2 U_{1}$ is the injection velocity at the solar wind velocity $U_{1}=400 \mathrm{~km} / \mathrm{s}$. Further, $n_{1}$ is the concentration of wind protons in the region of the shock wave of the heliosphere $\left(n_{1}=0.0005\right.$ $\left.\mathrm{cm}^{-3}\right), \varepsilon_{1} \cong 0.03$ is the fraction of protons reflected from the front and injected into the process of diffusive mechanism of acceleration by the shock, with the result of the particles of the ACR. In fact, as already noted, this fraction depends on the angles of inclination of the magnetic field and was determined in Fahr \& Versvharen (2008) (see below, on the alternative value of $\varepsilon_{1}$ ). The remaining quantities in (16) are the functions $\Lambda(s)=\left(\left[\frac{4 s-1}{3 s}-1\right] \frac{s-1}{3}\right)$ and $q=\frac{3 s}{s-1}$ with the shock compression ratio of $s=U_{1} / U_{2}$ Fahr \& Versvharen (2008).

Particle acceleration from the momentum value $p_{0}$ to $p_{\max }$ occurs in a characteristic time Drury (1983)

$$
\Delta \tau_{a c c}\left(p_{0}, p_{\max }\right)=\frac{3}{U_{1}-U_{2}} \int_{p_{0}}^{p_{\max }}\left(\frac{\kappa_{1}}{U_{1}}+\frac{\kappa_{2}}{U_{2}}\right) \frac{d p}{p},
$$

which for energies $E \leq 1 \mathrm{GeV}$ can be written as Fahr \& Versvharen (2008)

$$
\Delta \tau_{a c c}\left(p_{0}, p_{\max }\right)=\frac{3 \kappa_{0} \frac{v_{01}}{c}}{s-1} \frac{1+s}{U_{1} U_{2}}\left(x_{\max }-1\right) .
$$

Here the diffusion coefficient $\kappa$ is equal to

$$
\kappa=\kappa_{0} \frac{v_{01}}{c}\left(\frac{p}{p_{0}}\right)^{n}
$$

where $n=1$ for protons with $E \leq 1 \mathrm{GeV}$ and under the heliospheric conditions $\kappa_{0} \cong 10^{21}-10^{22} \mathrm{~cm}^{2} / \mathrm{s}$ Fahr \& Versvharen (2008), and references therein. Also, $x_{\max }=p_{\max } / p_{0}$ and $x_{0}=1$ are the upper and lower limits of integration in Eq. (17). The injection efficiency for transition to the acceleration mode, as is known, strongly depends on the number of seed particles reflected from the barrier in 
Table 1. The proton acceleration time $\Delta \tau_{a c c}$ for the indicated energies, and, for comparison, the characteristic diffusion time $\Delta \tau_{\text {diff }}$, for WR nebulae conditions (time in years, see text)

\begin{tabular}{lcc|cc|cc}
\hline$E=1 \mathrm{MeV}, \Delta \tau_{a c c}, \mathrm{yr}$ & $\Delta \tau_{\text {diff }}, \mathrm{yr}$ & $E=10 \mathrm{MeV}, \Delta \tau_{a c c}, \mathrm{yr}$ & $\Delta \tau_{\text {diff }}, \mathrm{yr}$ & $E=100 \mathrm{MeV}, \Delta \tau_{a c c}, \mathrm{yr}$ & $\Delta \tau_{\text {diff }}, \mathrm{yr}$ \\
\hline \multicolumn{7}{c}{} \\
\hline Time & & & & & \\
$s=2.31$ & 11 & 71298 & 36 & 22546 & 113 & 7130 \\
$s=3.00$ & 164 & 71298 & 517 & 22546 & 1636 & 7130 \\
$s=3.90$ & 860 & 71298 & 2719 & 22546 & 8600 & 7130 \\
\hline
\end{tabular}

the shock wave upstream and passing into diffusive acceleration mode. In Chalov \& Fahr (1996), the fraction of these particles was calculated (with an energy increase of about 30 times compared with the initial one), and using the distribution function of the mixture of wind protons with superthermal protons loaded upstream it was found that approximately $2 \%$ of all protons enter the acceleration mode resulting as ACR.

Here again the question arises of an analogy between shocks in the heliosphere and a WR (or PN) nebula, in particular, can one expect the presence of loaded particles in the region of shocks of WR nebulae, as in the heliosphere? The answer is positive, if we recall that the winds of WR stars, as well as stars at the previous stage, are very heterogeneous, including density condensations Smith (2014), which can serve as a source of seed injection of loaded particles. It should also be recalled that in Dworsky \& Fahr (2000) it was shown that, in reality, seed injection of loaded particles is not necessary, since it can be replaced by energy diffusion due to wave-particle interaction (Fermi-2 process), which again leads to the case with seed injection. Further, due to a more intense and faster stellar wind, and as a result, a higher density and possibly more developed turbulence in the WR nebulae as compared with the interstellar medium around the heliosphere, the conditions in these objects differ, which should affect the values of the diffusion coefficient. Indeed, according to the interpretation of gamma sources from supernova remnants interacting with close molecular clouds, the diffusion coefficients of particles in the energy range $\mathrm{GeV}-\mathrm{TeV}$ and higher are of the order of $10^{26}(E / 10 \mathrm{GeV})^{\delta} \mathrm{cm}^{2} / \mathrm{s}, \delta=0.5$ Aharonian \& Atoyan (1996). Obviously, for particles of lower energies, of the order of $\mathrm{MeV}$, the values of the diffusion coefficient should be less, therefore, taking the above estimate as the upper limit, we obtain an estimate of the upper limit of the acceleration time, according to Eq. (18). The results for different energies, for WR nebulae conditions are shown in Table 1. The corresponding diffusion times are also shown there according to the estimate $\Delta \tau_{\text {diff }}=L^{2} / 4 \kappa$ : the fact is that, for an obvious reason, the acceleration time cannot exceed the diffusion time in the acceleration region with a characteristic size $L$ Reimer et al. (2006) (here $L=r_{i}=3 \cdot 10^{18} \mathrm{~cm}$ ). Despite the roughness of the estimates used, it gives an idea of the order of the value of $\Delta \tau_{d i f f}$. As can be seen, the acceleration time in all cases is shorter than the diffusion time, with the exception of high-energy particles of about $100 \mathrm{MeV}$ at $s=$ 3.9. However, even at $E=50 \mathrm{MeV}$, again the acceleration time is less than the diffusion time for all $s$. Due to the steepness of the spectrum, this circumstance is not of particular importance in terms of radiation impact, for example, in radiation-chemical calculations a low energy part of the spectrum dominates, at most, in the range of 1-50 MeV. Finally, it should again be emphasized that Table 1 shows the upper limits of the acceleration time associated with the upper limits of the diffusion time used: for lower diffusion coefficients, the acceleration time decreases and the diffusion time increases. More accurate estimates of the diffusion time (as well as the diffusion coefficient) require self-consistent calculations using data on the magnetohydrodynamic turbulence spectrum and relative fluctuations of the magnetic field $\delta B / B$.

According to Fahr \& Versvharen (2008), the maximum achievable momentum value is estimated by the formula $(n=1)$ :

$$
p_{\text {max }} / p_{0}=\frac{c}{v_{02}} \sqrt{\frac{12}{q-4}\left[\frac{2+4 s+3 s_{2}}{3\left(1-s^{2}\right)}+\frac{q-2}{3}\right]+1},
$$

or $p_{\max } \cong 9 p_{0}, 119 p_{0}$ and $564 p_{0}$ respectively for $s=2.31,3.0$ and 3.9 . Then, as expected, $E_{\max } \cong$ 
947, 1877 and $7785 \mathrm{MeV}$ for the same $s$. Here it is necessary to distinguish $v_{01} \cong 2 U_{1}$ - the value of the initial injection velocity from $v_{02} \cong \sqrt{2 E_{02} / m}$ - the values at the start of the acceleration process, and, as already mentioned, $E_{02}=30 E_{01}$. Recall also that $U_{1}$ is $400 \mathrm{~km} / \mathrm{s}$ and $1500 \mathrm{~km} / \mathrm{s}$ for the solar wind, and for the WR star fast wind, respectively. Then, for the time of proton acceleration to the indicated energies, under the conditions of WR nebulae, the values given in Table 1 are obtained. From these data it follows that for a time shorter than the dynamic time of WR nebulae $\left(\sim 10^{5}\right.$ years $)$ part of the spectrum of energetic particles in the energy range $E \leq 100 \mathrm{MeV}$ it will have time to form (taking into account the above reservation regarding the exact value of the diffusion coefficient). It should be emphasized that with the expansion of the nebula, that is, with an increase in its inner radius, its ability to produce energy particles decreases, which follows from (16), especially if it is rewritten in the form Yeghikyan (2018b):

$$
\eta \frac{1}{4 \pi} \frac{\dot{M}_{f} V_{f}^{2}}{4 \pi r_{i}^{2}}=\int_{E_{1}}^{E_{\max }} \Phi_{E}(E) d E
$$

where $\dot{M}_{f}$ is the WR star's mass loss rate $(\mathrm{g} / \mathrm{s}), V_{f}$ is the wind speed $(\mathrm{cm} / \mathrm{s}), \Phi_{E}(E)$ is the differential intensity of accelerated particles (erg $\cdot \mathrm{cm}^{-2} \mathrm{~s}^{-1} \mathrm{sr}^{-1} \mathrm{MeV}^{-1}$ ), and $\eta$ is the fraction of the kinetic energy of the wind spent on the acceleration of protons by the shock wave (as in (16), i.e. here $\varepsilon \equiv \eta$ ) in energy range $E_{1} \leqslant E \leqslant E_{\max }$.

By the way, according to the generally accepted theory of the origin of galactic cosmic rays in the expanding shells of supernovae, usually $\eta=0.1-0.3$, but according to later numerical calculations supported by observations, larger values also are possible, $\eta=0.5$ Ellison et al. (2005).

In Fig. 2, the function $\Phi_{0}(E)$, which depends on different values of $s$, is shown for two values of $\varepsilon_{1}$ in the heliosphere, 0.03 and 0.0001 . It should be noted that according to heliospheric observations, $s_{o b s}=2.5$ Richardson et al. (2008).

It is interesting to compare the estimate of (16) for the solar wind with the measurements of the Voyager 1 probe Stone et al. (2005) (see their Fig. 2.). Assuming

$$
J_{E}(E)=J_{E}\left(E_{1}\right)\left(\frac{E_{1}}{E}\right)^{\gamma}, J_{p}(E)=\frac{J_{E}(E)}{E_{1}},
$$

where $E_{1} \mathrm{MeV}, \gamma=2-4$, for the differential particle intensity at $E=E_{1}=1 \mathrm{MeV}$ (in the mentioned units) we have Yeghikyan (2017b):

$$
J_{p}\left(E_{1}\right)=\frac{J_{E}\left(E_{1}\right)}{E_{1}}=\frac{\gamma-1}{E_{1}^{2}} \eta \frac{1}{4 \pi} \frac{\dot{M}_{f} V_{f}^{2}}{4 \pi r_{i}^{2}} .
$$

Taking $\dot{M}_{f}=2 \cdot 10^{-14} M_{\odot} / \mathrm{yr}, V_{f}=400 \mathrm{~km} / \mathrm{s}, r_{i}=100$ a.u., $\eta=0.1$, for $\gamma=2,3,4$ we obtain, respectively, $J_{p}\left(E_{1}\right) \cong 0.8,1.6,2.4$ particles $\cdot \mathrm{cm}^{-2} \mathrm{~s}^{-1} \mathrm{sr}^{-1} \mathrm{MeV}^{-1}$, which accuracies, up to a factor of 2 , coincide with the result of Voyager- 1 measurements, namely, in the same units, $\approx 1.4$ Stone et al. (2005), Scherer et al. (2008). In the same units, $\Phi_{02}=2.4$ for $s=2.31$ if $\varepsilon_{1}=0.0001$ is chosen. We emphasize once again that the phenomenological estimate (21) gives the same accuracy (within a factor of less than 2) if $\eta=0.1$ is chosen. A comparison of the functions of (16) and the estimate (21) is given in Fig. 2.

\section{Intensities of accelerated particles under conditions of WR and PN nebulae}

We now turn to the calculation of the differential intensity of energetic protons under the conditions of WR nebulae (see Fig. 3). Let take $\dot{M}_{f}=1 \cdot 10^{-5} M_{\odot} / \mathrm{yr}, V_{f}=1500 \mathrm{~km} / \mathrm{s}, r_{i}=3 \cdot 10^{18} \mathrm{~cm}, \eta=0.1$, then (17) give values, shown in Fig. 5, in comparison with (??) from Fahr \& Versvharen (2009), where the concentration of the fast wind near the shock is determined by the ratio $n_{1}=\dot{M}_{f} /\left(4 \pi r_{i}^{2} V_{f} m_{p}\right)$.

It should be emphasized that the average galactic value of the differential intensity for $\mathrm{CR}$ is $J_{p}\left(E_{1}\right)=2 \cdot 10^{-4}$ particles $\cdot \mathrm{cm}^{-2} \cdot \mathrm{s}^{-1} \cdot \mathrm{sr}^{-1} \mathrm{MeV}^{-1}$, that is, at the inner boundary of the nebula ( 1 or 


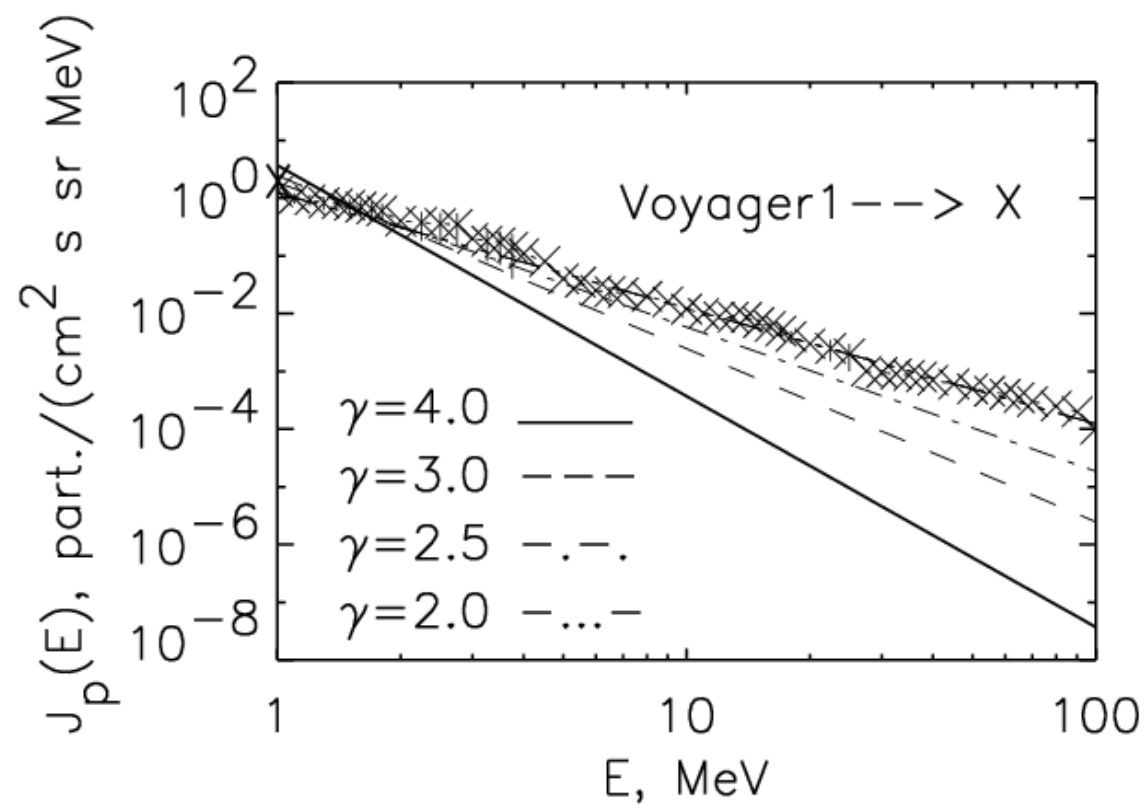

Figure 2. Results for the solar wind and corresponding Voyager 2 probe measurements

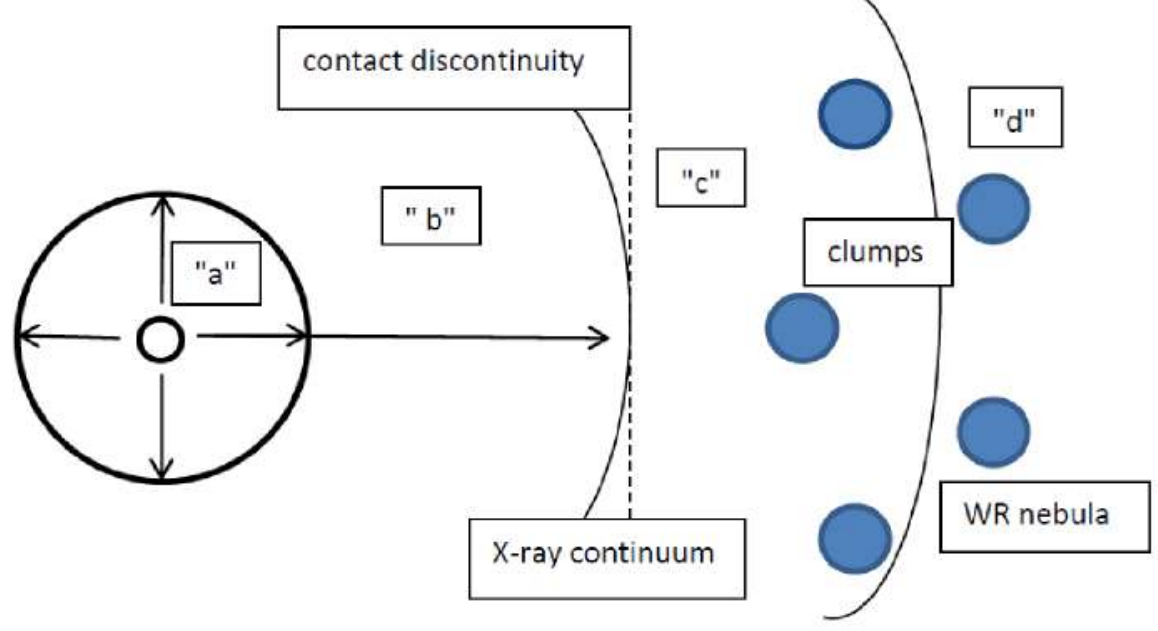

Figure 3. Schematic picture of the interaction of fast stellar wind with the circumstellar environment

$10 \mathrm{pc}$ ) the intensity of energetic particles (1000 or 10, respectively) by more than several orders of magnitude ( 7 or 5 ) exceeds the average galactic one. This is not surprising, since even the heliosphere, formed by a much weaker wind, nevertheless causes the appearance of similar accelerated particles by 4 orders of magnitude larger intensity than the average galactic Scherer et al. (2008).

Thus, WR (or PN) nebulae can be a very powerful local source of energetic particles (with not very high energies) and it would be interesting to study their possible observational manifestations in the nebula itself.

The first thing to be considered is the reason for the relatively low plasma temperature behind the shock emitting in the X-ray range. According to usual estimates, a flow at a speed of the order of $1500 \mathrm{~km} / \mathrm{s}$, colliding with a practically static (relative to the flow) matter, should cause a temperature value behind the shock of more than $10^{7} \mathrm{~K}$, while X-ray observations of WR nebulae using XMM and Chandra telescopes show a narrow range of values $1-2 \cdot 10^{6} \mathrm{~K}$ for diffuse sources Toala \& et al. (2017) and references therein. In other words, the theoretical post-shock temperature $T_{\text {shock }}=(3 / 16)(\mu / k) V_{f}^{2}$ (where $\mu \approx 0.6$ and 1.2 for a fully ionized matter with and without hydrogen, respectively) is more than an order of magnitude higher than the observed plasma temperature $T_{X}$ in the citet work. The authors of observations attribute such a decrease in temperature to the influence of electronic heat conduction. 


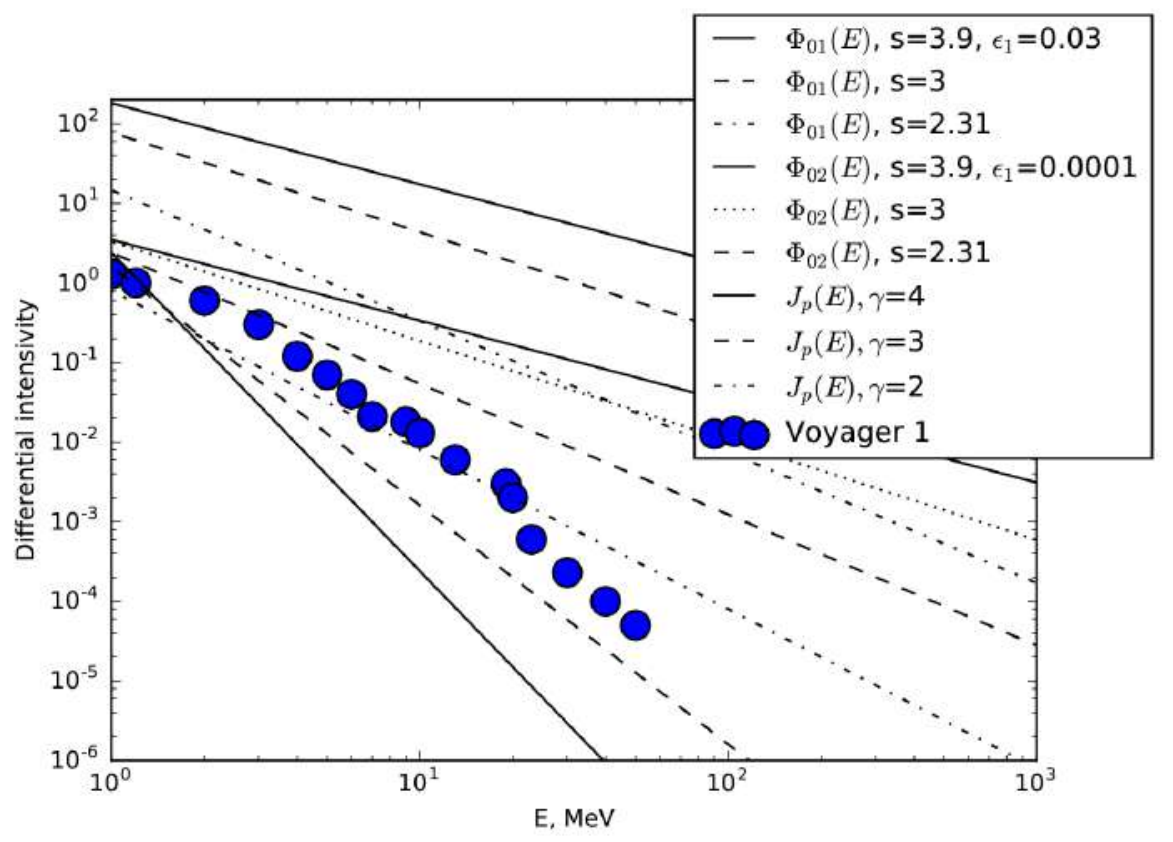

Figure 4. Differential intensities for various parameters of injection efficiency

It is interesting that the same phenomenon also occurs in the case of planetary nebulae Steffen et al. (2008), Toala \& Artur (2016) and references therein, where the same mechanism of heat conduction is discussed for explanation. Also, sometimes, the so-called loading ions coming from the condensation of matter and collided with fast wind were involved: before leaving the emitting X-ray radiation region, these ions are thermalized, lowering the temperature. However, there is no simple solution to this "low temperature problem" Soker \& et al (2010). It is clear that the solution of such problems should be based on a self-consistent consideration of the energy balance over all input and output channels, which is beyond the scope of this article. Therefore, without rejecting, in principle, mentioned possibilities, we recall that a similar problem in interpreting X-ray observations of supernova remnants is solved within the framework of the so-called theory of "diffusive acceleration by a modified shock wave" Decourchelle (2008) and references there. According to this theory, the thermal plasma behind the shock is heated to a temperature sufficient for radiation in the X-ray range, and at the same time, the shock itself accelerates the charged particles of the medium by a known diffusive mechanism, and changes the thermodynamic state of the medium: the more efficient the acceleration, the lower the temperature in the region of the shock wave Ellison et al. (2005), Decourchelle (2008). With effective acceleration, the above relationship between the speed of the fast flow and the steady-state temperature is no longer applicable, and must be consistent with the conditions of the modified shock wave. There is a lot of observational data of young supernova remnants illustrating this phenomenon and confirming the relationship between the effective acceleration of protons and the observed lower values of $T_{X}$ Ellison et al. (2005), Decourchelle (2008). Thus a small fraction of the kinetic energy of expansion of the shell spent on particle acceleration, at the same time, directly affects the X-ray radiation of the region around the shock wave. An analogy can be drawn between the expanding supernova shell in the interstellar medium (or in the medium of matter from the previous stellar wind) and the fast WR (or PN) stellar wind colliding with matter from the previous slow wind. Then, the lowered temperatures of the interaction region of the winds radiating in the $\mathrm{X}$-ray range can also be explained by the loss of energy for effective acceleration of protons, and leading to a decrease in temperature in the interaction region. Strictly speaking, the indicated (analytical) theory of diffusive acceleration by a modified shock wave Ellison et al. (2005), Decourchelle (2008) and references there, is developed for strictly relativistic particles, and quantitative criteria for the transition to the modification mode for the energy range 1-100 MeV are poorly known, and a separate consideration of the possibility of modification is required, which is beyond the scope of this work (see, however, below). Therefore, here we should confine ourselves to a qualitative statement of the fact that the lowered temperatures 


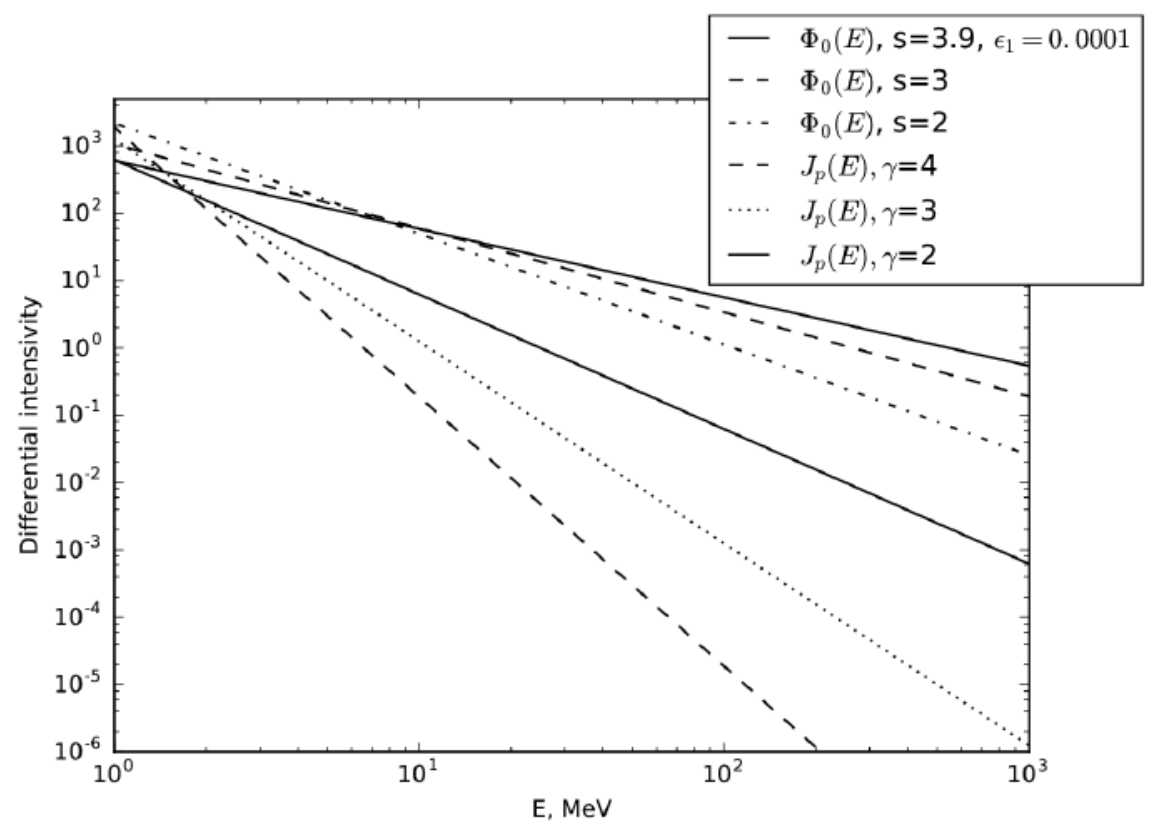

Figure 5. Differential intensities of energetic particles for WR nebulae

of the nebulae under consideration probably indicate effective acceleration by the shock wave, as, for example, in the case of supernova remnants.

Another aspect of the temperature problem behind the shock in the case of WR and PN nebulae is, depending on the situation, the different behavior of electrons and ions in the shock region, possibly also affecting the temperature. In this case, as the average electron energies in the region behind the shock wave in the heliosphere are of the order of $1 \mathrm{keV}$ Fahr et al. (2015), Fahr \& Verscharen (2016) that is, under similar shock wave conditions in WR nebulae (in turn, similar to the corresponding shock waves of planetary nebulae) for the temperature of electrons and protons one can write the following estimates Fahr et al. (2015), Fahr \& Verscharen (2016):

$$
T_{p}<T_{e} \lesssim \sqrt{\frac{m_{p}}{m_{e}}} T_{p} \simeq 43 T_{p}
$$

In conclusion of this section we emphasize once again that the shock modification mode, as described in Berezhko \& Ellison (1999), depends on a rather complicated way on the key parameters of the acceleration process, in particular, on such parameters as the momentum of injected particles proportional to $\lambda$ and the degree of injected particles $\eta$. $\lambda$ is determined by very complex processes $(\lambda>1)$, and for $\eta \geq \eta_{*}$, where

$$
\eta_{*}=\left(\frac{u_{0}}{c}\right)\left[\frac{2 \sqrt{50}}{3} \lambda\left(\frac{p_{\max }}{m c}\right)^{1 / 4}\right]^{-1}
$$

the shock is highly modified (here $u_{0}=V_{f}$ ). The critical value of the shock velocity $u_{0}^{*}$, corresponding to the transition to the modification mode is determined through the formula

$$
u_{0}^{*}=\frac{2 \sqrt{50}}{3} \lambda \eta\left(\frac{p_{\max }}{m c}\right)^{1 / 4} c
$$

and is equal (for $\lambda>1$ and for $p_{\max } / m c=1.39, E_{\max }=900 \mathrm{MeV}$ ) to the velocity $u_{0}^{*}=1500 \mathrm{~km} / \mathrm{s}$, regardless of the value of $\lambda$. The degree of particle injection in this case is $\eta_{*}=9.78 \cdot 10^{-4} / \lambda$. We emphasize that from the point of view of energy sources for chemical reactions, particles with energies of $1-10 \mathrm{MeV}$ are of the greatest interest, since even with minimal exponents $(\gamma \simeq 2.3)$, the steepness of the spectrum is still quite noticeable, and in the integrals describing, for example, the fraction of absorbed energy, particles with energies above 10-20 MeV practically do not contribute. Therefore, 
the formulas describing the energy spectra are merely illustrative, and when using them one should always remember the reservations with which they were obtained, especially in the case of relativistic particles.

Above, we obtained the value of the critical velocity of the shock transition to the modification mode, of the order of $1500 \mathrm{~km} / \mathrm{s}$, while for example, the observed values of terminal velocities for more than half of the sample of WR stars are more than $1500 \mathrm{~km} / \mathrm{s}$, (of the 50 observed WR stars, only 19 have the outflow velocity less than $1500 \mathrm{~km} / \mathrm{s}$, while for 31 are greater, reaching values of more than $2000 \mathrm{~km} / \mathrm{s}$ ) Niedzielski \& Skorzynski (2002). That is, for less than half of the WR stars, the shock waves caused by their stellar winds are not modified, and therefore, they cannot efficiently accelerate particles, and the pressure caused by them cannot be the reason for the relatively low temperature behind the front. At the same time, for the (larger) half of the (observed) WR stars, the positions of the above theory are quite applicable. This should be applicable also for PN cases where again similar distribution of outflow velocities exist. We note once again that in this article we are primarily interested in the energy distribution of particles in the immediate vicinity (10-20 MeV) from the lower edge of the spectrum of the order of $1 \mathrm{MeV}$.

\section{Dust irradiation with energetic particles}

Due to the high fluxes of energetic particles obtained, it is interesting to estimate the radiation doses of some species in such nebulae. We have expressions (??) for the flux of accelerated particles in the energy range 1-100 MeV, which is important in the irradiation of species, as well as in the ionization of molecular hydrogen. Assuming, as is mentioned above, the same parameters for WR and $\mathrm{PN}$, for the differential intensity of energetic protons at $1 \mathrm{MeV}$, we can write for two values of the internal radius $r_{i}=1-10 \mathrm{pc}$ :

$$
J_{p}\left(E_{1}\right)=10.0-1.0 \cdot 10^{3}
$$

in units particles $\cdot \mathrm{cm}^{-2} \cdot \mathrm{s}^{-1} \cdot \mathrm{sr}^{-1} \cdot \mathrm{MeV}^{-1}$, which by $5-7$ orders of magnitude larger than the average galactic one Scherer et al. (2008). Further, since the flux of energetic protons, the ionization rate $\zeta$, and the ionization cross section of molecular hydrogen by protons $\sigma$ are related by the simple relation $F(E) d E=\zeta / \sigma, F(E)=4 \pi J_{p}(E)$ Jenniskens (1993), it is clear that the ionization rate under such conditions will also be larger than the average value in the interstellar medium $\zeta(G C R): \zeta(W R)=$ $10^{5}-10^{7} \zeta(G C R)$. It is clear that such estimates make sense only when the WR nebula itself contains a molecular gas or is close enough to some molecular cloud, such as WR 7 (NGC 2359) Rizzo et al. (2001). In particular, for this nebula, the column density is of the order of $10^{20} \mathrm{~cm}^{-2}$ (ibid), which implies that protons with energies of $1 \mathrm{MeV}$ and higher will practically not lose energy along this path Yeghikyan (2017a), Yeghikyan (2017c), and the flow will decrease due to the divergence according to the law $r^{-2}$ Scherer et al. (2008). The influence of the magnetic field (if any) can be twofold: on the one hand protons can be reflected from magnetic inhomogeneities, and, depending on the strength and geometry of the magnetic field $(1-10 \mu G)$, the flux can decrease by 1-2 orders of magnitude Yeghikyan (2017c) and references therein. On the other hand, nonthermal protons of relatively low energies can possibly be accelerated to values of $1-10 \mathrm{MeV}$ in the presence of magnetohydrodynamic turbulence with a certain spectrum (Fermi-2 process) Cesarsky \& Volk (1978), Shchekinov (2005). Thus, in this case, assuming these processes compensate each other, the actual particle flux at the outer boundary of the nebula can decrease, at most, as $r^{-2}$, that is to the value of 10-1000 in the same units.

As is known dust is observed in many WR nebulae, the origin of which is still being discussed Hendrix (2016); possibly, dust is formed under the conditions of colliding winds of massive pairs Monnier et al. (2007) (in the case of PN dust is siply come with AGB winds).

It is possible to calculate the dose of dust irradiation $D_{p}$ by energetic protons in the nebula over time $t$ using a simple relation Yeghikyan (2017a) Yeghikyan (2017c) and references therein:

$$
n_{t} \cdot M\left(n_{t}\right) \cdot \frac{D_{p}}{d t}=\int_{E_{1}}^{E_{2}} F(E) S(E) d E, D_{p}=\frac{D_{p}}{d t} \cdot t,
$$

where $F(E)=4 \pi J_{p}(E)$ and $S(E)=-d E / d x$ is the energy losses of the particle during the passage of the path $d x$ (in units of $\mathrm{keV} / \mu$ ) in a dust particle with a concentration of $n_{t}$ and a molecular weight 
of $M\left(n_{t}\right)$ (accordingly, the amount of the absorbed by the dust particle energy $d E$ will be positive). $S(E)$ in the energy range 1-100 MeV can easily be calculated using the Bethe-Bloch formula, for example, using the SRIM computer program Ziegler et al. (2003), in particular, $S(E=1 \mathrm{MeV})=52.0$ $\mathrm{keV} / \mu$ for graphite with a density of $2.26 \mathrm{~g} / \mathrm{cm}^{3}\left(1.13\right.$ atom $\left./ \mathrm{cm}^{3}\right)$, and $S(E=10 \mathrm{MeV})=9.3 \mathrm{keV} / \mu$, while $S(E=50 \mathrm{MeV})=2.5 \mathrm{keV} / \mu$. For hydrogenated amorphous carbon (a: C-H) with a density of up to $2.4 \mathrm{~g} / \mathrm{cm}^{3}$ (Yeghikyan 2018 and references therein) the energy loss is approximately 1.06 times greater, $S(E=1 \mathrm{MeV})=55.1 \mathrm{keV} / \mu$. Further, choosing the least steep type of spectrum, $F(E)=F\left(E_{1}\right)\left(E_{1} / E\right)^{2}$ we obtain $D_{p}=1.5-150 \mathrm{eV} /$ a.m.u. for the values of the inner boundary about $r_{i}=10$ and $1 \mathrm{pc}$, respectively, and for a characteristic time interval of 100000 years. If there is a hydrogen deficiency in the stellar wind, that is, when helium predominates, the resulting value should be multiplied by 10, since the energy loss of particles is an order of magnitude larger, and the doses will be $15-1500 \mathrm{eV} /$ a.m.u.

In some cases, dust characteristic PAH emission bands are also observed Marchenko \& Moffat (2017). There is a point of view that further UV irradiation of PAH with subsequent dehydrogenation can even lead to the formation of fullerenes Scott \& et al. (1997), Otsuka \& et al. (2014), Zhen \& et al. (2014), which are observed in different (but WR radiation-like ) objects, for example, in planetary nebulae spectra Otsuka \& et al. (2014). Therefore, we also estimate the radiation dose for such systems (HAC, fullerenes) under conditions of WR nebulae, since, as a rule, in the radiation-chemical transformations of complex compounds, the electromagnetic and particle radiation are equivalent, and this fact must be taken into account Jenniskens (1993). From the point of view of irradiation of fullerenes, laboratory data on the stability of $\mathrm{C}_{60}$ and $\mathrm{C}_{70}$ are interesting: the oligomerization of molecular crystals with a dominant content of $\mathrm{C}_{60}$ (fullerite, density $1.7 \mathrm{~g} / \mathrm{cm}^{3}$ ) begins with radiation doses (either $\gamma$-rays or $\alpha$-particles are used) of the order $2.6 \mathrm{MGy}=2.6 \cdot 10^{10} \mathrm{erg} / \mathrm{g}=20 \mathrm{eV} /$ a.m.u., while amorphization requires about 100 times a larger dose of 250 MGy (2000 eV/a.m.u.). Cataldo \& et al. (2009). Thus, the dose of fullerite irradiation in the WR nebulae (at the inner boundary) is comparable to the dose for graphite (1.5-150 eV/a.m.u. for 100000 years), and for HAC it is somewhat larger. The doses of HAC decomposition and fragmentation of PAH are obviously less than laboratory values of the doses of fullerene amorphization and are comparable with the actual doses obtained in the WR nebulae, at which, as already noted, doses in the range of 1.5-150 eV/a.m.u. can be expected (15-1500 $\mathrm{eV} /$ a.m.u. in the case of hydrogen deficiency). Thus, not only dust can be formed in nebulae, but also PAH (which are observed in some cases), and even fullerenes, the manifestations of which should be looked for in the observed spectra.

\section{The formation of nitrogen oxides under the influence of CR in the atmospheres of earth's like exoplanets}

We now turn to the question of an excess of NO in the atmosphere (stratosphere) of exoplanets, using the Earth's atmosphere as an example. It has long been known that nitric oxide in the atmosphere, in addition to natural (purely terrestrial) sources, also has a formation channel due to CR, the contribution of which is usually small Chamberlain (1978). The mechanism of the process is as follows: CRs in the energy range $\mathrm{MeV}-\mathrm{GeV}$ and higher, penetrating the stratosphere, form a pair of ions for every $35 \mathrm{eV}$ of energy loss when interacting with air molecules. The process ends, ultimately, with the formation of nitrogen atoms $(\approx 1.4$ atoms from each pair of ions) that either destroy particles $\mathrm{NO}_{x}$

$$
\mathrm{N}+\mathrm{NO} \longrightarrow \mathrm{N}_{2}+\mathrm{O}+1.39 \mathrm{eV}, \beta_{29}=(8.2 \pm 1.4) \cdot 10^{-11} e^{-(460 \pm 60) / T} \mathrm{~cm}^{3} / \mathrm{s},
$$

or contribute to their formation,

$$
\mathrm{N}+\mathrm{O}_{2} \longrightarrow \mathrm{NO}+\mathrm{O}+1.39 \mathrm{eV}, \beta_{30}=5.5 \cdot 10^{-12} e^{3200 / T} \mathrm{~cm}^{3} / \mathrm{s} .
$$

If excited nitrogen atoms participate in the reaction, then the rate of the process via this channel is much higher:

$$
\mathrm{N}\left({ }^{2} \mathrm{D}\right)+\mathrm{O}_{2} \longrightarrow \mathrm{NO}+\mathrm{O}+3.76 \mathrm{eV}, \beta_{31}=7 \cdot 10^{-12} \mathrm{~cm}^{3} / \mathrm{s},
$$


since the activation energy of the reaction is mostly compensated by the excitation energy of the ${ }^{2} \mathrm{D}$ level of nitrogen atom. This is important because, according to estimates Chamberlain (1978), about half of the nitrogen atoms appear in an excited state. We briefly describe the formation of NO by reactions of $\mathrm{N}_{2} \mathrm{O}$ of a biospheric origin, with excited oxygen atoms (we indicate only those which are the most effective):

$$
\mathrm{O}\left({ }^{1} \mathrm{D}\right)+\mathrm{N}_{2} \mathrm{O} \longrightarrow 2 \mathrm{NO}+3.54 \mathrm{eV}, \beta_{32}=1.1 \cdot 10^{-10} \mathrm{~cm}^{3} / \mathrm{s} .
$$

The decay channels of $\mathrm{N}_{2} \mathrm{O}$ are collisions with the same exited oxygen in the stratosphere,

$$
\mathrm{O}\left({ }^{1} \mathrm{D}\right)+\mathrm{N}_{2} \mathrm{O} \longrightarrow \mathrm{N}_{2}+\mathrm{O}_{2}+5.4 \mathrm{eV}, \beta_{33}=0.7 \cdot 10^{-10} \mathrm{~cm}^{3} / \mathrm{s},
$$

and photodissociation, which is the main destruction mechanism:

$$
\mathrm{N}_{2} \mathrm{O}+\mathrm{h} \nu(\lambda \leqslant 2300 \mathrm{AA}) \longrightarrow \mathrm{N}_{2}+\mathrm{O}\left({ }^{1} \mathrm{D}\right) .
$$

So, the balance of $\mathrm{NO}_{x}$ in an unperturbed atmosphere depends on the formation rate of $\mathrm{N}_{2} \mathrm{O}$ at the Earth's surface, and the rate of its transfer to the stratosphere.

\section{concentration, $\mathrm{cm}^{-3}$}
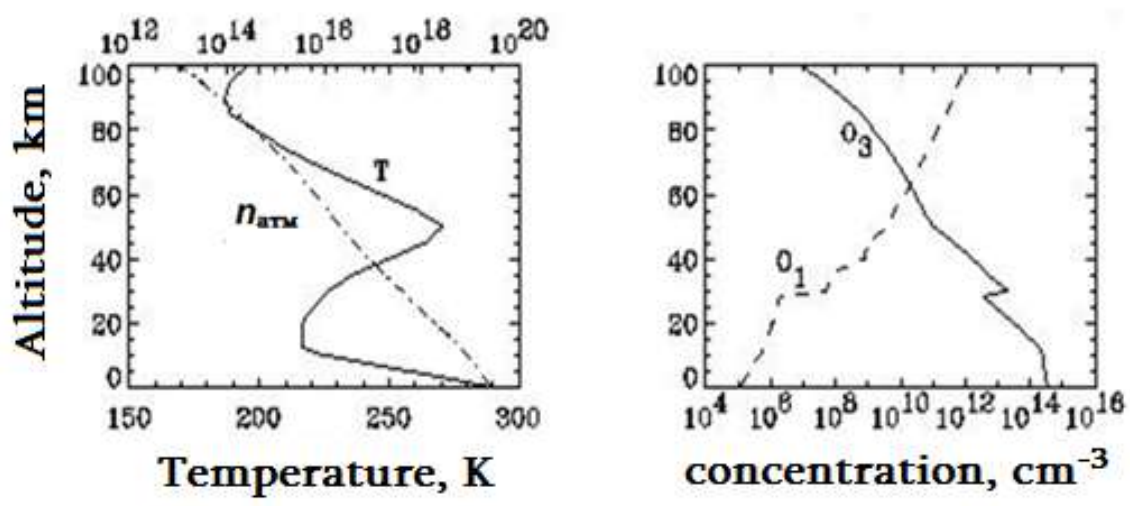

Figure 6. The standart atmospheric model of the atmosphere (left) and distibutions of atomar oxygen and ozone during a Chapman cycle (Chamberlain, 1978).

Result of the calculations can be summarized as follows. The ozone content in the perturbed atmosphere (and, for comparison, in the unperturbed one) was calculated according to the following scheme.

(P1) - Using US Standard Atmosphere data Brasseur \& Solomon (2005) on altitude profiles of average atmospheric gas concentrations and temperatures, we first calculate the distribution of atomic oxygen and ozone in the Chapman cycle approximation Chamberlain (1978), that is without $\mathrm{HO}_{x}$ and $\mathrm{NO}_{x}$ (see Fig. 6). Recall that hereinafter we assume the presence of photochemical equilibrium, which in the lower stratosphere, $(z \leqslant 25-30 \mathrm{~km})$, is not satisfied, and implies that the distribution of ozone in this region also depends on the dynamic processes of vertical and horizontal mixing of air masses Chamberlain (1978).

Indeed, according to observations, the calculated ozone concentrations at these altitudes are approximately 30-40\% higher than those observed Chamberlain (1978). In part, this difference is explained by the contribution of the impurities $\mathrm{HO}_{x}, \mathrm{NO}_{x}$, and $\mathrm{ClO}_{x}$ the first two will be taken into account below. The remainder of the ozone deficit is apparently responsible for industrial emissions of fluorocarbons (although there is a problem explaining the transfer to the stratosphere), methane, biospheric methane, and volcanic gases, the exact accounting of which is possible only within the framework of a non-stationary dynamic physicochemical model. As for the phenomenon of the socalled "ozone holes" (ozone deficit of about 30-40\%, relative to the average annual value) observed in the circumpolar regions, they are seasonal in nature, and there is a consensus of researchers that they can be explained by the effect of a full combination of the above factors, within, of course, 

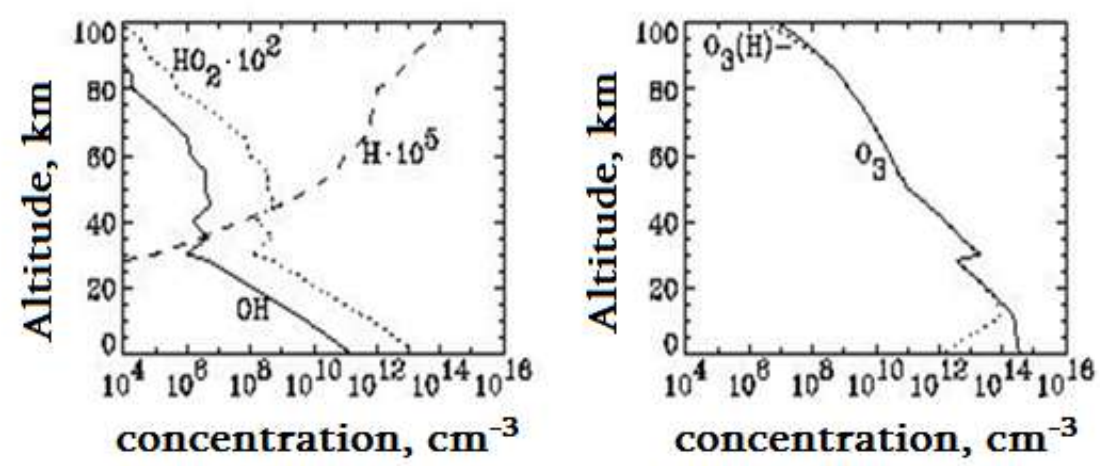

Figure 7. Distributions of $\mathrm{HO}_{x}$ (left), influence on ozone (right)

self-consistent 3-dimensional dynamic models. The effect of the excess of $\mathrm{HO}_{x}$ and $\mathrm{NO}_{x}$, caused by extraterrestrial factors considered in this paper, causes much larger changes in ozone, which makes it possible to use the above (semi)analytical model.

(P2) - We now take into account the influence of $\mathrm{HO}_{x}$ in an undisturbed atmosphere, for which we use their altitude profiles known from observations Chamberlain (1978). The calculation results are shown in Fig. 7. As one can see, a slight effect is noticeable only in the upper mesosphere $(80-100) \mathrm{km}$, and in the layers adjacent to the troposphere $(z \leqslant 20 \mathrm{~km})$, and in the latter case, the result cannot be considered correct, due to the lack of equilibrium conditions.

(P3) - The influence of $\mathrm{NO}_{x}$ is taken into account as is mentioned above, the results are presented in Fig. 8. Note that here the $\mathrm{NO}_{x}$ profiles are calculated according to the data from Chamberlain (1978), Crutzen et al. (1975), and, as already noted, it is the inclusion of $\mathrm{N}_{2} \mathrm{O}$ that allows one to adjust the calculated profiles with the average observables. It is interesting that the contribution from the current galactic CR (GCR) flux (although insignificant) can both increase the ozone content (in the upper stratosphere) and lower it (between the troposphere and stratosphere). Recall that in the latter case, this (analytical) model may be incorrect, and that accounting for (small) contributions from small impurities is currently implemented by numerical dynamic models, involving all possible mechanisms. Similarly, the solar CR (SCR) spectrum, caused by the powerful solar flare of 1972, reduces the ozone content in the upper stratosphere (also slightly). The energy spectrum of cosmic rays in the heliosphere is given in Yeghikyan (2018a). Recall that the anomalous CR (ACR) component increases when the Sun passes through the ISM clouds (in the range of 1-100 MeV), in particular, at $n(H) \sim 100 \mathrm{~cm}^{-3}$, the intensity of ACR will increase by about $10^{3}$ times, assuming that in the modern era, in the vicinity of the Sun, $n(H) \sim 100 \mathrm{~cm}^{-3}$. Assuming that the concentrations of $\mathrm{NO}_{x}$ will increase by the same amount, we use these (extrapolated) values to calculate the ozone content when the Earth passes through a diffuse HI cloud. The calculation results are shown in Fig. 9

As one can see, due to passing through a different $\mathrm{HI}$ cloud, with $n(H) \sim 100 \mathrm{~cm}^{-3}$, the $\mathrm{O}_{3}$ abundance at heights of $20 \mathrm{~km} \leq z \leq 60 \mathrm{~km}$ decreases by 1-3 orders of magnitude (!). Recall that through such clouds the Sun passes about 10 times more often than through MC (every 30 million years), and reduced values of $n\left(O_{3}\right)$ must be maintained in the atmosphere over a period of time of several hundred thousand years. Obviously, such a decrease in the concentration of ozone in the stratosphere should be accompanied by significant changes in climate, and as a result of the increased background of UV radiation, a catastrophic restructuring of environmental relations. The consequences of the so-called superflares on the Sun are also quite large - a 10-fold excess of the 1972 flare intensity Crutzen et al. (1975), can reduce the ozone content in the stratosphere by no less than an order of magnitude (Fig. 11, right).

(P4) - The influence of $\mathrm{HO}_{x}$ increased due to the possible passage of the Sun through the MC with $n(H) \sim 1000 \mathrm{~cm}^{-3}$ and higher, we take into account, according to Yeghikyan \& Fahr (2004). The calculation results for $n(H) \sim 10^{3}-10^{5} \mathrm{~cm}^{-3}$, are shown in Fig. 11. As expected, a decrease of $n\left(O_{3}\right)$ is significant in the mesosphere, which qualitatively coincides with the result obtained above (Fig. 10). The quantitative difference reaches a factor of $2-3$, and is apparently associated with the more 

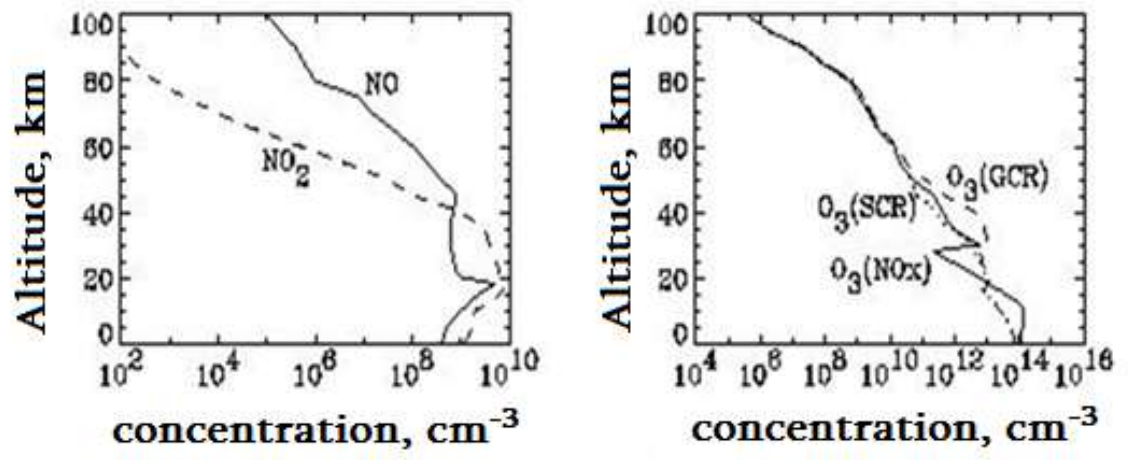

Figure 8. Distributions of $\mathrm{NO}_{x}$ when the influence of $\mathrm{N}_{2} \mathrm{O}$ dominates (left), influence on ozone: $\mathrm{O}_{3}\left(\mathrm{NO}_{x}\right)$ - contribution of only $\mathrm{N}_{2} \mathrm{O}, \mathrm{O}_{3}(\mathrm{GCR})$ - contribution of GCR, $\mathrm{O}_{3}(\mathrm{SCR})$ - contribution of the solar flare caused a tenfold increase of SCR (right).

refined approach used here to take into account the influence of $\mathrm{HO}_{x}$. In this case, the effect is more pronounced, and therefore, the conclusion made on the formation of a global layer of nocticlucent clouds in the mesosphere, with subsequent serious climatic consequences, remains valid.
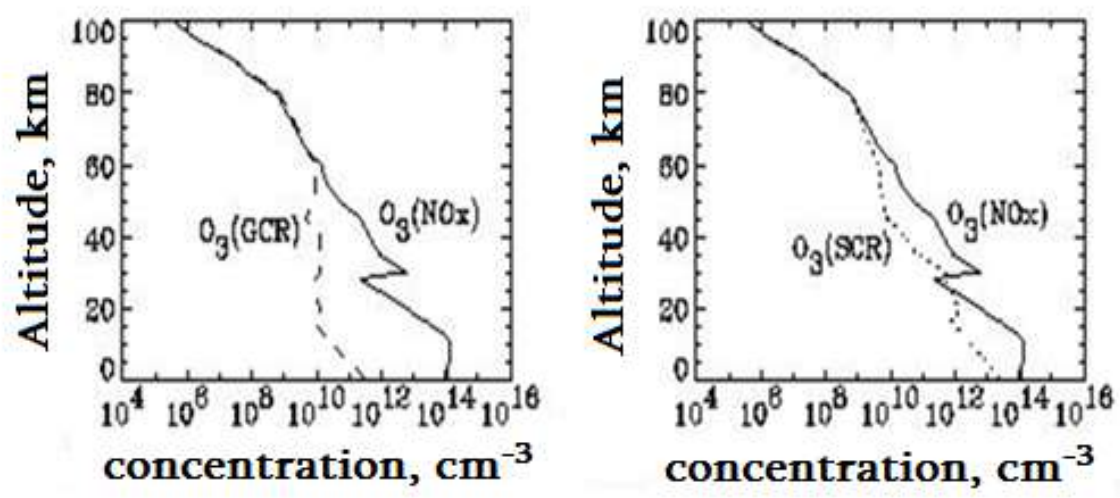

Figure 9. Ozone content with increased CR fluxes. $\mathrm{O}_{3}\left(\mathrm{NO}_{x}\right)$ - unperturbed value, $\mathrm{O}_{3}(\mathrm{GCR})$ - values taking into account ACR induced by the cloud with $n(\mathrm{H}) \sim 100 \mathrm{~cm}^{-3}, \mathrm{O}_{3}(\mathrm{SCR})$ - values taking into account a tenfold intensity of solar CR.

(P5) - The total effect of the increased, due to the passage of the Sun through the $\mathrm{MC} \mathrm{HO}_{x}$ and $\mathrm{NO}_{x}$, was taken into account by a combination of the listed above two items (P3, P4), for the case of passing through the MC with a concentration of $n(H) \sim 1000 \mathrm{~cm}^{-3}$. The result is shown in Fig. 11 .

\section{Conclusion}

This article reviewed the role of low-energy cosmic rays (CR) with energies of $1 \mathrm{MeV}$ and higher in astrophysics. Processes of establishing the ionization balance in dence interstellar clouds, the timescale of star formation by collapsing clouds and the role of ambipolar diffusion are considered.

Particulat attention is paid to the description of mechanisms of particle acceleration at the MHD wave front. A semi-analytical particle acceleration formula is proposed depending on the star mass loss rate, outflow velocity and the wind kinetic energy conversion factor to the particle acceleration energy. In the solar wind case this formula coincides remarkably with the Voyager 2 zond observations at the mentioned conversion factor to be of $10 \%$.

Irradiation by particles in the $\mathrm{MeV}-\mathrm{GeV}$ energetic range of species like $\mathrm{HAC}, \mathrm{PAH}$ and fullerens, as well as water ice and other ice mixtures, is also described. 

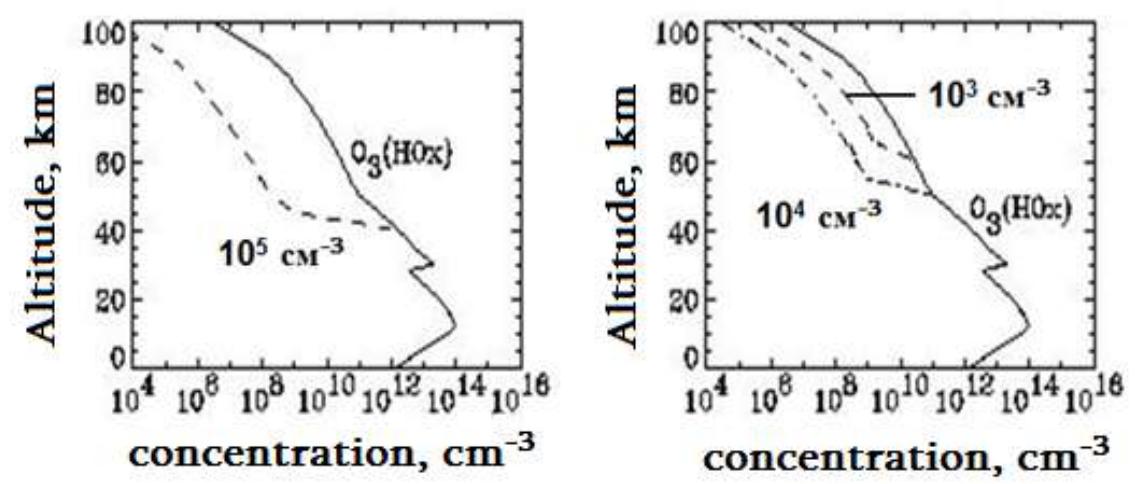

Figure 10. The ozone abundance at the increased fluxes of $\mathrm{H}$ and ACR particles caused by the Sun passing through interstellar clouds with concentration of $n(\mathrm{H}) \sim 10^{3}, 10^{4}$ and $10^{5} \mathrm{~cm}^{-3}$.

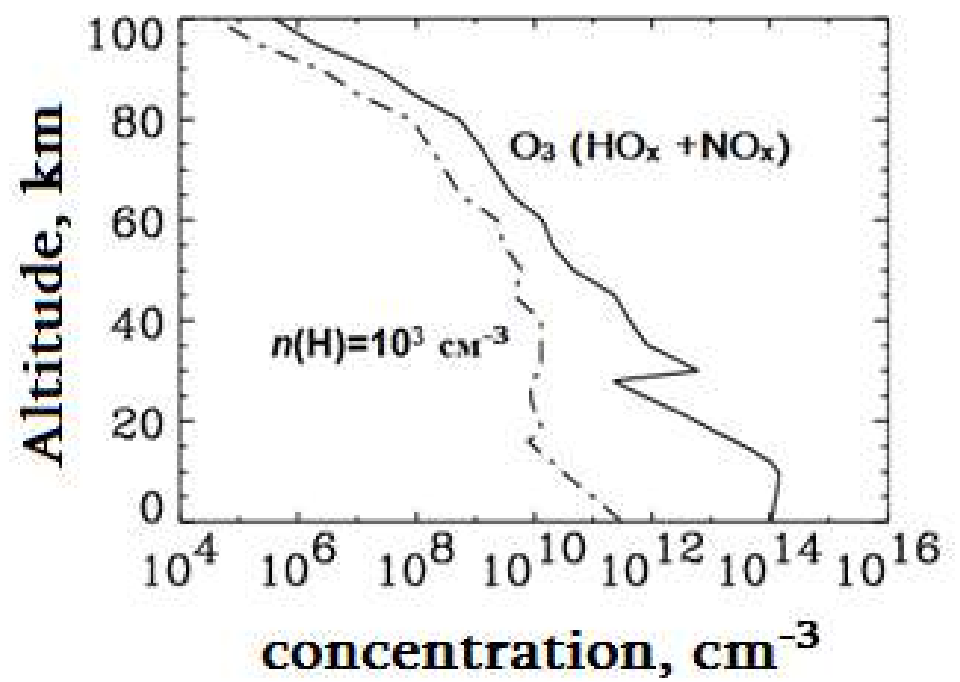

Figure 11. The ozone abundance at the increased fluxes of $\mathrm{H}$ and ACR particles caused by the Sun passing through interstellar clouds with concentration of $n(\mathrm{H}) \cdot \mathrm{O}_{3}\left(\mathrm{HO}_{\mathrm{x}}+\mathrm{NO}_{\mathrm{x}}\right)-$ is the unperturbed value of $\mathrm{O}_{3}$.

The question of the formation of nitric oxide under the influence of cosmic rays in the atmosphere of exoplanets is also considered on the example of the terrestrial atmosphere. The ozone content in both the mesosphere and the stratosphere decreases with the passage of the Earth through the MC. The impact on the mesosphere is associated with the penetration of the hydrogen atoms of the cloud into the atmosphere, and the increased at the orbit of the Earth's ACR flux in the range of 1-100 MeV causes an increase in $\mathrm{NO}_{x}$, which, in turn, affects the ozone content in the stratosphere and causes climate changes.

\section{Acknowledgements}

The author acknowledge the partial support of this work by Ambartsumian research award in 2019-2020. 


\section{References}

Aharonian Atoyan 1996, Astronom. Astrophys., 309, 917

Bartelmann M., 2013

Berezhko E., Ellison D., 1999, Astrophys. J., 526, 385

Bodenheimer P., 2011, Priciples of Star Formation

Brasseur G., Solomon S., 2005, Aeronomy of the Middle Atmosphere

Bykov A., Ellison D., Marcowith A., Osipov S., 2018, Space Science Review, 214, 41

Cataldo F., et al. 2009, MNRAS, 394, 615

Cesarsky C., Volk H., 1978, Astron. Astrophys., 70, 367

Chalov S., Fahr H., 1996, Solar Physics, 168, 369

Chamberlain J., 1978, Theory of planetary atmospheres

Crutzen P., Isaksen I., Reid G., 1975, Science, 189, 457

Decourchelle A., 2008, Mem. S.A.It., 79, 44

Diehl R., Halloin H., Kretschmer K., et al. 2006, Nature, 439, 45

Dopita M., Sutherland R., 2003, Astrophysics of the Diffuse Universe

Drury 1983, Rep. Prog. Physics, 46, 973

Dworsky A., Fahr H., 2000, Astronom. Astrophys., 353, L1

Ellison D., Decourchelle A., Ballet J., 2005, Astron. Astrophysics, 429, 569

Fahr H., Verscharen D., 2016, Astron. Astrophys., 587, L1

Fahr H., Versvharen D., 2008, Astron. Astrophysics, 487, L21

Fahr H., Versvharen D., 2009, Astrophys. Space Sci. Trans., 5, 21

Fahr H., Richardson J., Verscharen D., 2015, Astron. Astrophys., 579, A18

Ginzburg V. L., 1996, Physics-Uspekhi, 166, 1108

Hendrix T., 2016, MNRAS, 460, 3975

Jenniskens P., 1993, Astron. Astrophys., 273, 583

Longair M., 2011, High Energy Astrophysics

Marchenko S., Moffat A., 2017, MNRAS, 468, 2416

Mathis J., Mezger P., Panagia N., 1983, Astron. Astrophys., 128, 212

Monnier et al. M., 2007, Astrophys. J., 655, 1033

Niedzielski A., Skorzynski W., 2002, Acta Astronomica, 52, 81

Otsuka M., et al. 2014, MNRAS, 437, 2577

Padmanabhan T., 2000, Theoretical Astrophysics I

Reimer A., Pohl M., Reimer O., 2006, Astrophys. J., 644, 1118 
Richardson J., Kasper J., Wang C., et al. 2008, Nature, 454, 63

Rizzo J., Martin-Pintado J., Mangum J., 2001, Astronom. Astrophys., 366, 146

Scherer K., Fahr H., 2009, Astron. Astrophys., 495, 631

Scherer K., Fichtner H., Ferreira S., et al. 2008, Astrophys. J., 680, L105

Schlickeiser R., 2002, Cosmic Ray Astrophysics

Scott A., et al. 1997, Astrophys. J, 489, L193

Shchekinov Y., 2005, Astron. Rep., 49, 269

Smith N., 2014, Ann. Rev. Astron. Astrophys., 52, 487

Soker et al 2010, Astrophys. J, 725, 1910

Steffen M., Schoenberner D., Warnuth A., 2008, Astron. Astrophys., 489, 173

Stone E., Cummings A., McDonald F., et al. 2005, Science, 309, 2017

Tielens A., 2005, The Physics and Chemistrz of the Interstellar Medium

Toala J., Artur S., 2016, MNRAS, 463, 4438

Toala J., et al. 2017, Astrophys. J., 846, 76

Yeghikyan A., 2017a, Astrophysics, 54, 87

Yeghikyan A., 2017b, Astrophysics, 60, 374

Yeghikyan A., 2017c, ISRN Astronomy and Astrophysics, 2011, id.905015

Yeghikyan A., 2018a, Physics and Astronomy International Journal, 2, 149

Yeghikyan A., 2018b, Astrophysics, 469, 469

Yeghikyan A., Fahr H., 2004, Astron. Astrophys., 425, 1113

Zhen J., et al. 2014, Astrophys. J., 797, L30

Ziegler J., Biersack J., Littmark U., 2003, The Stopping and Range of Ions in Solids 